$\mathrm{p}<0.001)$. Post-hoc analysis shows that the antrum was better visualised compared to all other gastric stations $(\mathrm{p}<0.001)$. When composite scores of fundus, antrum and upper body are compared to the distal body, incisura and antrum, views in the distal half of the stomach are significantly better visualised $\left(\chi^{2}=520, p=0.006\right)$. Procedural distress, AD and AP were $4.9( \pm 0.5), 2.8( \pm 0.5)$ and $1.3( \pm 0.3)$ respectively: none correlated with views in any station apart from those with more procedural $\mathrm{AD}$ who had poorer views of the distal gastric body $(\mathrm{r}=-0.36 \mathrm{p}=0.017)$. Sedation had no effect on distress, discomfort or pain or views of any station. Procedure time was $5 \mathrm{~m} 41 \mathrm{~s}( \pm 18)$ with $55 \mathrm{~s}( \pm 5)$ spent in retroflexion and $58 \mathrm{~s}( \pm 9)$ in the duodenum, none of which correlated with any visualisation scores. Time spent in the duodenum correlated with increasing procedural AP $(r=0.415, p=0.009)$. Patients $(25 \%)$ who said they would decline repeat OGD reported significantly greater AP $(3.0 \pm 0.9$ vs $0.7 \pm 0.3)$ and procedural distress $(7.7 \pm 0.5$ vs $4.0 \pm 0.6)$, but their visualisation scores, procedural times or use of sedation did not differ from those who would have a repeat test.

Conclusion Visualisation at OGD is variable, with excellent antral but comparatively poorer proximal gastric and incisura views. OGD is the cause of significant distress to patients, rather than discomfort or pain, although duodenal examination may be painful in some. There was little correlation of quality of views with sedation, tolerance or duration of examination.

\section{PTH-061 MISSED OESOPHAGOGASTRIC CANCER CORRELATES WITH HIGHER LIST INTENSITY BUT NOT RATES OF SEDATION}

David FW Tai* ${ }^{*}$ Andrew Hopper, Mark McAlindon. Sheffield Teaching Hospitals NHS Trust, Sheffield, UK

\subsection{6/gutjnl-2018-BSGAbstracts.82}

Introduction Oesophagogastric (OG) cancers diagnosed within three years of an unremarkable oesophagogastroduodenoscopy (OGD) can be considered a failure to earlier diagnose the OG cancer, or post-OGD upper GI cancer (POUGIC). Retrospective studies suggest that they comprise up to $11 \%$ of OG cancer diagnosis [Menon, Endosc Int Open 2014] and auditing rates of POUGIC is a recent quality standard for endoscopy units [Beg, Gut 2017]. We examined whether patient sedation or procedural burden affects the rate of POUGIC.

Methods Cases of OG cancer diagnosed at OGD between Jan 2013 and Dec 2016 at Sheffield Teaching Hospitals were identified from our upper GI cancer database. OGD performed up to three years prior to diagnostic OGD were reviewed to identify cases of POUGIC. Rates of sedation and number of procedural points (one for OGD; two for colonoscopy; plus one for therapeutics) on lists were compared in three groups: a) the index procedures, b) the diagnostic procedures and c) age, sex and endoscopist matched patient control procedures in which focal (mucosal or vascular) lesions (FL) were identified. FL were approximated in size and location (oesophageal or gastric) as a surrogate for an early neoplastic lesion.
Results A total of 553 patients $(64.2 \%$ male, mean age $72 \pm 1$, $50.4 \%$ gastric) were diagnosed with OG cancer. Forty $(7.2 \%$, mean age $74 \pm 2,55 \%$ male, $55 \%$ gastric,) patients had 47 non-diagnostic procedures up to three years prior to diagnosis. Mean time from index to diagnostic OGD was $486 \pm 55$ days. In $42.5 \%$ OGD was performed within one year of diagnosis. There was no difference in the age, gender and rates of sedation (25 vs $28.5 \%$ ) between patients at index and diagnostic procedures. At index OGD the sedation rates were higher (44.7 vs $26.3 \% \mathrm{p}=0.049$ ) than at diagnostic OGD but there was a greater number of procedural points on the list $(7.9$ vs $9.3 \mathrm{p}=0.007)$. Control patients $(\mathrm{n}=38$, mean age $72 \pm 2$ $\mathrm{p}=0.64$ compared to POUGIC patients) with FL had OGD done a median of 33 days ( -357 to 728 days) from the index OGD. No suitable controls were identified in 2 patients. There was no difference in the sedation rates $(25.0$ vs $26.3 \% \mathrm{p}=0.89$ ) but there was a trend towards a higher number of procedural points $(9.3$ vs $8.7 \mathrm{p}=0.057)$ between the index OGD for POUGIC patients and their controls.

Conclusions The local POUGIC rate is $7.2 \%$. No differences in sedation rate between index, diagnostic or control procedures with representative FL suggest use of sedation may not help detection of early neoplastic lesions. However, endoscopy lists with OGDs which miss OG cancer seem to have a heavier burden than ones that diagnosed cancer and other FL suggesting that reduced list intensity may reduce the likelihood of missed pathology.

\section{PTH-062 CLINIC BASED OUTPATIENT TRANSNASAL ENDOSCOPY:IMPLEMENTATION AND EVALUATION OF AN INNOVATIVE ENDOSCOPY SERVICE}

${ }^{1}$ Nilanjana Tewari ${ }^{*},{ }^{1}$ Adolfo Parra-Blanco, ${ }^{1}$ Sarmad Sami, ${ }^{1}$ Shiv Budihal, ${ }^{1}$ Nina Lewis, 1James Catton, ${ }^{1,2}$ Krish Ragunath. ${ }^{1}$ Nottingham University Hospitals NHS Trust, Nottingham, UK; ${ }^{2}$ Nottingham Digestive Diseases BRU, Nottingham, UK

\subsection{6/gutjnl-2018-BSGAbstracts.83}

Introduction There is increasing evidence that Transnasal endoscopy (TNE), performed with an ultrathin HD scope, is well tolerated with minimal cardiorespiratory stress and better patient experience than standard endoscopy. We report preliminary results from a new outpatient TNE service developed in a university teaching hospital which is a tertiary referral centre for gastroenterology and upper gastrointestinal surgery.

Methods After local governance approvals, TNE was introduced and performed by 5 experienced endoscopists. All procedures were performed in an outpatient clinic adjacent to the endoscopy recovery area over a 6 month period. Patients were assessed as suitable for TNE based on local guidelines and if agreeable, underwent TNE using Pentax EPK-i7000 HD video endoscopy processor and EG16-K10 Transnasal endoscope (outer diameter $5.4 \mathrm{~mm}, 2.0 \mathrm{~mm}$ instrument channel) under topical anaesthetic plus anti-congestant applied to nostril. An antifoam/mucolytic drink was given $15 \mathrm{~min}$ prior to procedure. If the nose could not be intubated, the patient was offered the procedure using the narrow endoscope trans-orally. Preliminary data was collected in a pilot study in which patients were asked to complete a visual analogue score (VAS) and 
questionnaire. Data on all patients undergoing TNE was collected prospectively and retrospectively analysed from the hospital computer records.

Results Since its introduction, 113 patients have been assessed as suitable for TNE. 67 females and 46 males (median age 62, IQR 52.5-70 years) underwent TNE. The first 17 patients were part of the pilot study. Of 96 subsequent patients, 66 were direct to test referrals on the cancer pathway, 10 other referrals on the cancer pathway, 13 routine, 3 planned surveillance, 1 urgent and 3 urgent inpatients. The most common indications were dysphagia (55 patients) and dyspepsia (36 patients). Endoscopy was completed trans-nasally in 92 patients $(81.4 \%)$, trans-orally in 16 patients $(14.1 \%)$ and failed in 5 patients $(4.4 \%)$. Reasons for performing trans-orally were narrow nasal passages in 7 patients, 2 patients on warfarin with high INR, 2 patient choice and 7 didn't tolerate scope in nose. Duodenal intubation was successful in 107/113 (94.7\%). There were no abnormal findings in 57 patients, inflammation was seen in 36 patients and 8 cancers of the oesophagus/oesophago-gastric junction were found. Biopsies were taken in 63 procedures and all were adequate for histology. Procedures were tolerated well with no immediate complications. Median (range) VAS was $9(5-10)$ and of those who had had previous OGD, 71\% expressed a preference for TNE and 29\% preferred neither.

Conclusions TNE delivered in an outpatient clinic setting with immediate access to endoscopy unit is a safe and effective method of investigating upper gastrointestinal tract symptoms. This innovative service delivery has the potential to reduce traditional diagnostic gastroscopy and increase capacity.

\section{PTH-063 ENDOSCOPIC RESECTION OF DYSPLASTIC LESIONS IN COLITIS}

${ }^{1}$ Asma Al-Khandari”, $\quad{ }^{1}$ Sreedhari Thayalasekaran, ${ }^{2}$ Megha Bhandari, ${ }^{1}$ Kesavan Kandiah Hayee, ${ }^{3}$ Marek Bugajski, ${ }^{3}$ Agnieszka Przybysz, ${ }^{4}$ Michal Spychalski, ${ }^{5}$ Bu Hayee, ${ }^{6}$ Alessandro Repici, ${ }^{1}$ Pradeep Bhandari. 'Queen Alexandra Hospital, Portsmouth, UK; ${ }^{2}$ Cambridge University, Cambridge, UK; ${ }^{3}$ Maria Sklodowska-Curie Institute Oncology, Warsaw, Poland; ${ }^{4}$ General Hospital, Brzeziny, Poland; ${ }^{5}$ Kings College Hospital," UK; ${ }^{6}$ Humanitas Research Hospital, Milan, Italy

\subsection{6/gutjil-2018-BSGAbstracts.84}

Introduction Cumulative colon cancer risk is estimated at $2 \%-$ $18 \%$ depending on duration of colitis. Management of flat neoplasia in colitis remains controversial. BSG guidelines recommend colectomy if complete endoscopic resection isn't guaranteed. Aim of this study was to assess need for surgery in the management of flat neoplasia in colitis.

Methods A multicentre cohort study of all flat neoplasia endoscopically resected in colitis in 5 tertiary European centres from 2008-2017.

Results 101 flat neoplasia were resected in 85 patients at 5 European centres. Mean age 61 years (range 28-82). Mean size of lesions $34 \mathrm{~mm}$ (range $8-120 \mathrm{~mm}$ ).

\begin{tabular}{|c|c|c|c|c|c|}
\hline & KAR & KAR & EMR & EMR Rectum & Total \\
\hline & Colon & Rectum & Colon & (8) & (101) \\
\hline & (26) & (13) & (54) & & \\
\hline Recurrence & 2 & 0 & 5 & 0 & 7 \\
\hline Complications & 5 & 0 & 2 & 0 & 7 \\
\hline En-bloc & 14 & 11 & 33 & 5 & 63 \\
\hline
\end{tabular}

\begin{tabular}{|c|c|c|c|c|c|}
\hline & $\begin{array}{l}\text { KAR } \\
\text { Fibrosis } \\
(26)\end{array}$ & $\begin{array}{l}\text { KAR No fibrosis } \\
\text { (13) }\end{array}$ & $\begin{array}{l}\text { EMR } \\
\text { Fibrosis } \\
(15)\end{array}$ & $\begin{array}{l}\text { EMR No fibrosis } \\
\text { (47) }\end{array}$ & $\begin{array}{l}\text { Total } \\
(101)\end{array}$ \\
\hline Recurrence & 1 & 1 & 0 & 5 & 7 \\
\hline Complications & 5 & 0 & 1 & 1 & 7 \\
\hline En-bloc & 15 & 10 & 8 & 30 & 63 \\
\hline
\end{tabular}

Abstract PTH-063 Table 3 Lesion size

\begin{tabular}{llllll}
\hline & KAR & KAR & EMR & EMR & Total \\
& $0-$ & $>20 \mathrm{~mm}$ & $0-$ & $>20 \mathrm{~mm}$ & \\
& $20 \mathrm{~mm}$ & & $20 \mathrm{~mm}$ & & \\
\hline Recurrence & 0 & 2 & 3 & 2 & 7 \\
Complications & 0 & 5 & 1 & 1 & 7 \\
En-bloc & 4 & 21 & 37 & 1 & 63 \\
\hline
\end{tabular}

$40 \%$ of the lesions were treated by KAR. There was no difference in lesion location between EMR and KAR. Lesions $>20 \mathrm{~mm}$ in size were removed more by KAR than EMR. More lesions removed by KAR (26) had fibrosis compared to EMR (15). 7 complications occurred in the cohort; 3 cases of bleeding and 4 perforations. Bleeding was controlled endoscopically. 3 perforations were managed endoscopically and 1 required surgery. 7/86 (8.1\%) lesions with follow up data had recurrence.

Multi-variate regression analysis concluded;

- 33. EMR leads to higher recurrence rates, irrespective of size, location and fibrosis ( $\mathrm{p}$-value of 0.048 )

- 34. KAR leads to higher complication rates in the colon as compared to rectum ( $\mathrm{p}$-value of 0.045 )

- 35. KAR shows a trend towards better en-bloc resection ( $\mathrm{p}-$ value 0.063 ).

5 lesions underwent surgery; 3 due to cancer; 1 due to perforation; 1 due to failure of endoscopic resection. Histology; 88 adenoma (low-grade dysplasia), 6 adenoma (high-grade dysplasia), 3 cancers and 4 sessile serrated polyps.

Conclusions This is the largest reported cohort of endoscopic resection of flat neoplasia in colitis. We demonstrate that both KAR and EMR are feasible in colitis with only 5\% of patients requiring surgery. Fibrosis is very common in colitis. Recurrence is higher with EMR and complications higher with KAR. Our data shows that lesions with fibrosis are best treated by KAR, and those with fibrosis and $<20 \mathrm{~mm}$ can be managed by EMR.

\section{PTH-064 ANATOMICAL FEATURES OF THE ILEOCAECAL JUNCTION AND THEIR IMPLICATIONS FOR PROOF OF COLONOSCOPY COMPLETION}

${ }^{1}$ Ruth Townend*, ${ }^{2}$ Amanda Choo, ${ }^{2}$ Eunice Wong, ${ }^{1}$ Cecilia Brassett, ${ }^{1,3}$ Jonathan Brown. ${ }^{1}$ Human Anatomy Teaching Group, Department of PDN, Cambridge, UK; ${ }^{2}$ University of Cambridge School of Clinical Medicine, UK; ${ }^{3}$ Gloucestershire Hospitals NHS Foundation Trust, UK

\subsection{6/gutjnl-2018-BSGAbstracts.85}

Introduction A completion rate for colonoscopy in excess of $90 \%$ is a Joint Advisory Group quality assurance standard; 